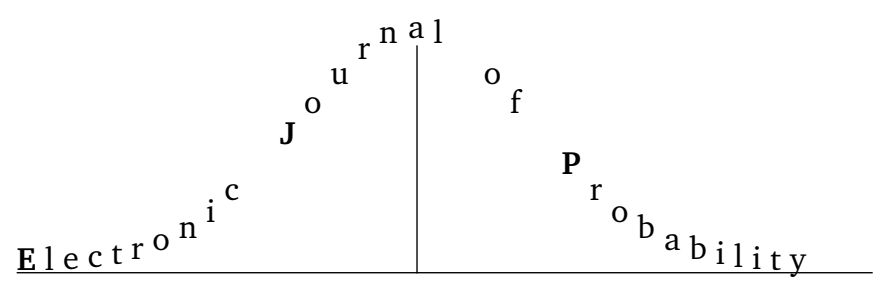

Vol. 14 (2009), Paper no. 51, pages 1513-1531.

Journal URL

http://www.math.washington.edu/ ejpecp/

\title{
Lower estimates for random walks on a class of amenable $p$-adic groups
}

\author{
Sami Mustapha \\ Institut mathématique de Jussieu \\ 175, rue du Chevaleret, 75013, Paris, France \\ sam@math.jussieu.fr
}

\begin{abstract}
We give central lower estimates for the transition kernels corresponding to symmetric random walks on certain amenable $p$-adic groups.
\end{abstract}

Key words: random walk; $p$-adic groups.

AMS 2000 Subject Classification: Primary 22E35, 60B15, 60G50.

Submitted to EJP on May 5, 2005, final version accepted April 3, 2009. 


\section{Introduction}

Let $G$ be a locally compact amenable group. We shall denote by $d^{r} g$ (resp. $d^{l} g$ ) the right (resp. left) Haar measure on $G$ and by $\delta(g)=d^{r} g / d^{l} g$ the modular function on $G$ normalized by $\delta(e)=1$ where $e$ denotes the identity of $G$. let $d \mu(g)=\varphi(g) d^{r} g \in \mathbf{P}(G)$ be a probability measure on $G$ where $\varphi(g) \in L^{\infty}(G)$ is assumed to have a compact support or a fast decay at infinity. Let us assume that $\mu$ is symmetric (i.e. the involution $g \rightarrow g^{-1}$ stabilizes $\mu$ ) and consider the random walk on $G$ induced by $\mu$, i.e. the $G$-valued process that evolves as follows: if $X_{n}=g$ is the position at time $n$ then $X_{n+1}=g h$ where $h$ is chosen according to $\mu$. We shall denote by

$$
d \mu^{* n}(g)=\varphi_{n}(g) d^{r} g
$$

the $n^{\text {th }}$ convolution power of $\mu$ and examine the behaviour of the decay of $\varphi_{n}(e)$ as $n \rightarrow \infty$.

If we restrict ourselves to unimodular real Lie groups then the answer lies in the behaviour of the volume growth of $G$. Let us recall that if $G$ is a locally compact group that is generated by some symmetric compact neighbourhood $\Omega \subset G$ of the identity in $G$, the volume growth function is (cf. [9])

$$
\gamma(t)=\operatorname{Vol}\left(B_{t}(e)\right), \quad t=1,2, \ldots
$$

where the volume is taken with respect to $d^{r} g$ (or $d^{l} g$ ) and where $B_{t}(e)$ is the ball of radius $t$ centred on $e$ defined by

$$
B_{t}(e)=\Omega \ldots \Omega, \quad t \text { times. }
$$

For $x \in G$ the distance from $e$ is defined by $|x|=\inf \left\{t, x \in B_{t}(e)\right\}$ and a left invariant distance can be defined on $G$ by setting $d(x, y)=\left|y^{-1} x\right|, x, y \in G$. If $\Omega_{1}, \Omega_{2}$ are two neighbourhoods of $e$ as above it is not difficult to check that there exists $C>0$ such that $C^{-1} \leq|\cdot|_{2} /|\cdot|_{1} \leq C$ and that the corresponding growth functions satisfy the obvious equivalence $\gamma_{1} \approx \gamma_{2}$, i.e.

$$
\gamma_{1}(t) \leq C \gamma_{2}(C t)+C \leq C^{\prime} \gamma_{1}\left(C^{\prime} t\right)+C^{\prime}, \quad t \geq 1
$$

For real Lie groups we have the following dichotomy (cf. [9], [13]): either

$$
\gamma(t) \approx t^{D}
$$

where $D=D(G)=1,2, \ldots$, or

$$
\gamma(t) \approx e^{t}
$$

In the first case we say that $G$ is of polynomial growth and in the second case we say that $G$ is of exponential growth and the answer to our problem in the case of unimodular amenable real Lie groups was given by Varopoulos and is the following

$$
\begin{aligned}
& \varphi_{n}(e) \approx n^{-D / 2} \Longleftrightarrow \gamma(t) \approx t^{D} \\
& \varphi_{n}(e) \approx e^{-n^{1 / 3}} \Longleftrightarrow \gamma(t) \approx e^{t}
\end{aligned}
$$

(cf. [1], [6], [7], [10], [28]).

Varopoulos showed that the $e^{-n^{1 / 3}}$ versus polynomial behaviour extends to the non-unimodular amenable case depending on wether the Lie group $G$ is $(C)$ or $(N C)$. This classification introduced 
in [24] can be expressed in terms of the roots of the ad-action of the radical of the Lie algebra of the group on its nilradical.

The discret case is more complicated (cf. [1], [11], [16], [17], [22], [23]). First there is no dichotomy in the volume growth (cf. [8]). On the other hand if we suppose that the group $G$ is of exponential growth then one can claim only the upper bound (cf. [11])

$$
\varphi_{n}(e) \leq C \exp \left(-c n^{1 / 3}\right), \quad n \geq 1 .
$$

In general, the matching lower bound fails. Ch. Pittet and L. Saloff-Coste showed (cf. [16]) that there are soluble groups with exponentional volume growth for which the heat kernel decays as $\exp \left(-c n^{\alpha}\right)$ with $\alpha \in(0,1)$ which can be taken arbitrarly close to 1 . This, as mentionned above, can not happen in the case of real Lie groups.

In a recent paper (cf. [17]) Ch. Pittet and L. Saloff-Coste established the lower bound $\varphi_{2 n}(e) \geq$ $c \exp \left(-C n^{1 / 3}\right), n=1,2, \ldots$ for the large times asymptotic behaviours of the probabilities of return to the origin at even times $2 n$, for random walks associated with finite symmetric generating sets of solvable groups of finite Prüfer rank. They asked in this paper (cf. [17], §8) if a similiar lower bound is available in the case of analytic $p$-adic groups. An answer to this problem was given in [15] (cf. also [14]). The aim of this paper is to show that the $e^{-n^{1 / 3}}$ lower bound obtained in [14] can be substantially improved for a large class of amenable $p$-adic groups.

\section{Amenable $p$-adic groups}

In this section $G$ will denote an algebraic connected amenable group over $\mathbb{Q}_{p}$ the field of $p$-adic numbers; $U \subset Q \subset G$ will denote the radical and the unipotent radical (cf. [3], [5]). Amenability of $G$ is equivalent to the fact that the semi-simple group $G / Q$ is compact (cf. [19]). Let $S$ denote a fixed levi subgroup $S$ of $G$ (cf. [3]). The group $G$ can then be written as a semi-direct product:

$$
G=Q \ltimes S=(U \ltimes A) \ltimes S \cong U \ltimes(A \times S)
$$

where $A$ is abelian and can be identified to the direct product of a finite group and a $d$-torus $T \cong$ $\left(\mathbb{Q}_{p}^{*}\right)^{d}$ (cf. [3], [5]). Here $\mathbb{Q}_{p}^{*}$ denotes the multiplicative group of the field $\mathbb{Q}_{p}$. Since $\left(\mathbb{Q}_{p}^{*}\right)^{d} \cong \mathbb{Z}^{d} \times K$ (where $K$ is compact, cf. [2], [4]), by considering the projection

$$
\pi: A \longrightarrow \mathbb{Z}^{d},
$$

we can fix $\pi_{1}, \ldots, \pi_{d} \in A$ so that each $z \in A$ admits a unique decomposition

$$
z=\pi_{1}^{n_{1}} \ldots \pi_{d}^{n_{d}} \tau, \quad n_{1}, \ldots, n_{d} \in \mathbb{Z}, \quad \tau \in \tilde{K},
$$

where $\tilde{K}$ denotes a compact subgroup of $A$. Let $\mathscr{U}=\operatorname{Lie}(U)$ denote the Lie algebra of $U$. Let $\overline{\mathbb{Q}}_{p}$ denotes a finite extension of $\mathbb{Q}_{p}$ which contains all the eigenvalues defined by

$$
\operatorname{det}\left(\operatorname{Ad}\left(\pi_{j}\right)-\lambda I\right)=0, \quad j=1, \ldots, d .
$$

The $A d$-action of $A$ on $\mathscr{U}$ extends to $\mathscr{U} \otimes_{\mathbb{Q}_{p}} \overline{\mathbb{Q}}_{p}$ and it follows from the proof of the Zassenhaus lemma (cf. [12]) that we have a decomposition of $\mathscr{U} \otimes_{\mathbb{Q}_{p}} \overline{\mathbb{Q}}_{p}$ into a direct sum

$$
\mathscr{U} \otimes_{\mathbb{Q}_{p}} \overline{\mathbb{Q}}_{p}=W_{1} \oplus \ldots \oplus W_{r}
$$


where the subspaces $W_{j}(1 \leq j \leq r)$ are invariant by $A d\left(\pi_{i}\right), i=1, \ldots, d$, and such that the restriction of $A d\left(\pi_{i}\right)$ to $W_{j}$ is the sum of the scalar $\lambda_{j}\left(\pi_{i}\right)$ and a nilpotent endomorphism.

Let $\chi_{j}: A \longrightarrow \overline{\mathbb{Q}}_{p}^{*}$ defined by

$$
\chi_{j}(z)=\lambda_{j}\left(\pi_{1}\right)^{n_{1}} \ldots \lambda_{j}\left(\pi_{d}\right)^{n_{d}}, z=\pi_{1}^{n_{1}} \ldots \pi_{d}^{n_{d}} \tau, j=1, \ldots, r
$$

Let $|.|_{p}$ denote the standard $p$-adic norm. We shall denote by $|.|_{p}^{\prime}$ its extension to $\overline{\mathbb{Q}}_{p}$. We have $|x|_{p}^{\prime} \in\left\{\bar{p}^{n}, n \in \mathbb{Z}\right\} \cup\{0\}, x \in \overline{\mathbb{Q}}_{p}$, where $\bar{p}$ denotes a rational power of the prime $p$ (cf. [2]). Let $\alpha_{1}, \alpha_{2}, \ldots, \alpha_{s}$ denote the different norms of the $\chi_{j}$ 's, i.e. the different homomorphisms obtained by considering $A \longrightarrow \bar{p}^{\mathbb{Z}}, z \longrightarrow\left|\chi_{j}(z)\right|_{p}^{\prime}, j=1, \ldots, r$. Let $\mathscr{L}=\left\{\alpha_{1}, \alpha_{2}, \ldots, \alpha_{s}\right\}$. We shall assume that $\mathscr{L}$ is nonempty and that

$1 \notin \mathscr{L}$.

Here 1 denotes the homomorphism $A \rightarrow \bar{p}^{\mathbb{Z}}$ identically equal to 1 . This assumption garantees the fact that the group $G$ is compactly generated (cf. [3]). Observe that the group $G$ is then of exponential volume growth (cf. [18]).

Let $\gamma_{j, 1}, \ldots, \gamma_{j, d} \in \mathbb{Z}$ the integers defined by

$$
\alpha_{j}(z)=\bar{p}^{\gamma_{j, 1} n_{1}+\gamma_{j, 2} n_{2}+\ldots+\gamma_{j, d} n_{d}}, \quad z=\pi_{1}^{n_{1}} \ldots \pi_{d}^{n_{d}} \tau, j=1, \ldots, s .
$$

We shall denote by $L_{j}, j=1, \ldots, s$, the linear forms on $\mathbb{Z}^{d}$ defined by

$$
L_{j}\left(n_{1}, \ldots, n_{d}\right)=\gamma_{j, 1} n_{1}+\gamma_{j, 2} n_{2}+\ldots+\gamma_{j, d} n_{d}
$$

$\left(n_{1}, \ldots, n_{d}\right) \in \mathbb{Z}^{d}$ and by $\tilde{L}_{j}$ the linear forms on $\mathbb{R}^{d}$ induced by the $L_{j}$ 's.

Let now $d \mu(g)=\varphi(g) d^{r} g \in \mathbf{P}(G)$ denote a symmetric probability measure on $G$. The density $\varphi(g)$ is assumed to be a continuous compactly supported function on $G$. To avoid unnecessary complications we shall assume that there exists $e \in \Omega=\Omega^{-1} \subset G$ such that

$$
\inf \{\varphi(g), \quad g \in \Omega\}>0, \quad G=\bigcup_{n \geq 0} \Omega^{n}
$$

the last condition in (7) implies that $\operatorname{supp}(\mu)$ generates the group $G$.

Let

$$
p: G \longrightarrow G / U \longrightarrow A
$$

denote the projection that we obtain from the identifications (1) and let

$$
\check{\mu}=(\pi \circ p)(\mu) \in \mathbf{P}\left(\mathbb{Z}^{d}\right) \subset \mathbf{P}\left(\mathbb{R}^{d}\right),
$$

where $\pi$ denotes the canonical projection (2). It follows from (7) that there exists a choice of coordinates on $\mathbb{R}^{d}$ for which the covariance matrix of $\breve{\mu}$ satisfies

$$
\int_{\mathbb{R}^{d}} x_{i} x_{j} d \check{\mu}(x)=\delta_{i, j}, \quad 1 \leq i, j \leq d .
$$

We shall assume that $\mathbb{R}^{d}$ is equipped with the Euclidean structure associated to these coordinates. 
From now on we shall assume that the $L_{j}$ 's induce a "Weyl chamber". More precisely we suppose that

$$
\Pi_{\mathscr{L}}=\left\{x \in \mathbb{R}^{d}, \quad \tilde{L}_{j}(x)>0, \quad j=1,2, \ldots, s\right\} \subset \mathbb{R}^{d}
$$

define a nonempty convex cone in $\mathbb{R}^{d}$. This condition is the analogue of the (NC)-condition introduced by Varopoulos in [24] in the setting of real amenable Lie groups. We shall prove that, under this condition, we have a lower bound of the form $\varphi_{n}(e) \geq c n^{-v}$. The argument follows the approach introduced by Varopoulos in [24].

The exact value of $v$ is defined as in the real case and is expressed in terms a parameter $\lambda=\lambda(d, \mathscr{L})$ that is defined as follows. In the the rank one case (i.e. $d=1$ ) we shall set

$$
\lambda=0 .
$$

In the case $d \geq 2$ let us denote by $\Sigma=\left\{x \in \mathbb{R}^{d},|x|=1\right\}$ the unit sphere in $\mathbb{R}^{d}$. Let $\Delta_{\Sigma}$ be the corresponding spherical Laplacian. Let $\Pi_{\Sigma}=\Sigma \cap \Pi_{\mathscr{L}} \subset \Sigma$. We then set

$$
\lambda=\inf \left\{-\left(\Delta_{\Sigma} f, f\right),\|f\|_{2}=1, f \in C_{0}^{\infty}\left(\Pi_{\Sigma}\right)\right\}
$$

i.e. $\lambda$ is the first Dirichlet eigenvalue of the region $\Pi_{\Sigma}$. The scalar product and the $L^{2}$-norm in $\left(10^{b}\right)$ are taken with respect to the Euclidean volume element on $\Sigma$.

Theorem 1. Let $G$ and $d \mu^{* n}(g)=\varphi_{n}(g) d^{r} g, n=1,2, \ldots$ be as above. Then there exists $C>0$ such that

$$
\varphi_{n}(e) \geq \frac{1}{C n^{1+\frac{\sqrt{(d-2)^{2}+4 \lambda}}{2}}}, \quad n=1,2, \ldots
$$

where $\lambda$ is defined by (10).

The following comments may be helpful in placing the above theorem in its proper perspective.

(i) It is enough to to prove the estimate (11) when $n$ is even since $\varphi$ satisfies (7).

(ii) Observe that the group $G$ is automatically non-unimodular, for otherwise we have (cf. [11]):

$$
\varphi_{n}(e) \leq C e^{-c n^{1 / 3}}, \quad n=1,2, \ldots
$$

(iii) The upper estimate

$$
\varphi_{n}(e) \leq \frac{C}{n^{3 / 2}}, \quad n=1,2, \ldots
$$

is known to hold for general non-unimodular locally compact groups (cf. [26]). This shows that the index $3 / 2$ cannot be improved in the rank one case.

(iv) We will show (cf. $\S 4$ below) that in the case of metabelian $p$-adic groups, the lower bound (11) can be complemented with a similar upper bound.

Throughout the remainder of the paper $C$ denotes a positive constant which is not always the same, even in a given line. 


\section{Proof of Theorem 1}

Let $G, d \mu(g)=\varphi(g) d^{r} g \in \mathbf{P}(G)$ and $d \mu^{* n}(g)=\varphi_{n}(g) d^{r} g$ be as in Theorem 1. Let $\xi_{1}, \xi_{2}, \ldots \in G$ be a sequence of independent equidistribued random variables of law $d \mu(g)$ and let $X_{n}=\xi_{1} \xi_{2} \ldots \xi_{n}$, $n=1,2, \ldots$ denote the corresponding random walk starting at $X_{0}=e$. Let $B \subset G$ a borel subset, we have

$$
\mathbb{P}_{e}\left[X_{n} \in B\right]=\int_{B} \varphi_{n}(g) d^{r} g, n=1,2, \ldots
$$

The symmetry of $d \mu(g)$ implies that

$$
d \mu(g)=d \mu\left(g^{-1}\right)=\varphi(g) d^{r} g=\varphi\left(g^{-1}\right) d g=\varphi\left(g^{-1}\right) \delta(g)^{-1} d^{r} g,
$$

hence

$$
\varphi\left(g^{-1}\right)=\varphi(g) \delta(g), \quad g \in G .
$$

We have also

$$
\varphi_{n}\left(g^{-1}\right)=\varphi_{n}(g) \delta(g), g \in G, \quad n=1,2, \ldots
$$

On the other hand we have

$$
\varphi_{2 n}(e)=\int_{G} \varphi_{n}\left(g^{-1}\right) \varphi_{n}(g) d g=\int_{G} \varphi_{n}(g) \delta(g) \varphi_{n}(g) d g=\int_{G} \varphi_{n}(g)^{2} d^{r} g .
$$

Schwarz inequality applied to (12) gives then

$$
\varphi_{2 n}(e) \geq \frac{\left(\mathbb{P}_{e}\left[X_{n} \in B\right]\right)^{2}}{|B|}, \quad B \subset G, \quad n=1,2, \ldots
$$

where $|B|$ denotes the right Haar measure of $B$.

Let us further observe that the group $G$ (resp. $G / U$ ) decomposes as a semi-direct (resp. direct) product

$$
\begin{gathered}
G=Q \ltimes S \cong U \ltimes\left(\mathbb{Z}^{d} \times \tilde{S}\right) \\
G / U \cong A \times S \cong \mathbb{Z}^{d} \times \tilde{S}
\end{gathered}
$$

where $\tilde{S}$ is compact. This follows from (1).

Let us write

$$
X_{n}=\xi_{1} \xi_{2} \ldots \xi_{n}=u_{1} z_{1} u_{2} z_{2} \ldots u_{n} z_{n}, \quad n=1,2, \ldots
$$

where $\xi_{j}=u_{j} z_{j}$ with $u_{j} \in U$ and $z_{j} \in A \times S, j=1,2, \ldots$. Using the interior automorphisms $x^{y}=$ $y x y^{-1}, x, y \in G$, we rewrite (14)

$$
\begin{gathered}
X_{n}=u_{1} u_{2}^{z_{1}} u_{3}^{z_{1} z_{2}} \ldots u_{n}^{z_{1} \ldots z_{n-1}} z_{1} z_{2} \ldots z_{n}=\Gamma_{n} Z_{n}, \\
\Gamma_{n} \in U, \quad Z_{n} \in A \times S, \quad n=1,2, \ldots
\end{gathered}
$$


We shall use the exponential map and identify $U$ to its Lie algebra $\mathscr{U}$ (cf. [5], [20]) and write each $u_{j}$ in the above expression

$$
u_{i}=\exp \left(v_{i}\right), \quad v_{i} \in \mathscr{U}, \quad i=1,2, . .
$$

We have therefore

$$
u_{j}^{z_{1} z_{2} \ldots z_{j-1}}=\exp \left(\operatorname{Ad}\left(z_{1} \ldots z_{j-1}\right) v_{j}\right), \quad j \geq 2 .
$$

Let us fix $e_{1}, e_{2}, \ldots, e_{m}$ a basis of $\mathscr{U} \otimes_{\mathbb{Q}_{p}} \overline{\mathbb{Q}}_{p}$ adapted to the decomposition (4). For $x=x_{1} e_{1}+x_{2} e_{2}+$ $\ldots+x_{m} e_{m} \in \mathscr{U} \otimes_{\mathbb{Q}_{p}} \overline{\mathbb{Q}}_{p}$, we set

$$
\|x\|=\max _{1 \leq i \leq m}\left|x_{i}\right| .
$$

Since $\mu$ is compactly supported we can suppose that the $v_{i}$ 's in (16) satisfy

$$
\left\|v_{j}\right\| \leq C, \quad j=1,2, \ldots
$$

where $C>0$ is an appropriate positive constant.

Let us equip $E n d_{\overline{\mathbb{Q}}_{p}}\left(\mathscr{U} \otimes_{\mathbb{Q}_{p}} \overline{\mathbb{Q}}_{p}\right)$ with the norm

$$
\|\mid T\|=\sup _{\|v\| \leq 1}\|T v\|, \quad T \in \operatorname{End}_{\overline{\mathbb{Q}}_{p}}\left(\mathscr{U} \otimes_{\mathbb{Q}_{p}} \overline{\mathbb{Q}}_{p}\right) .
$$

It is clear that ||$||||$. satisfies the ultrametric property

$$
\left\|T+T^{\prime}\right\| \mid \leq \max \left(\left\|\left|T\|\mid,\| T^{\prime}\|\|\right) .\right.\right.
$$

Let $1 \leq l \leq r$. For

$$
z=\pi_{1}^{n_{1}} \ldots \pi_{d}^{n_{d}} \tilde{\tau}=\tilde{z} \tilde{\tau} \in A \times S \cong \mathbb{Z}^{d} \times \tilde{S}
$$

we have

$$
\left.\operatorname{Ad}(z)\right|_{W_{l}}=\left.\operatorname{Ad}(\tilde{\tau})\right|_{W_{l}} \circ\left(\tilde{\chi}_{l}(z) I+\mathscr{T}(z)\right),
$$

where $\mathscr{T}(z)$ denotes an upper triangular matrice and where

$$
\tilde{\chi}_{l}(z)=\chi_{l}\left(\pi_{1}^{n_{1}} \ldots \pi_{d}^{n_{d}}\right)=\chi_{l}\left(\pi_{1}\right)^{n_{1}} \ldots \chi_{l}\left(\pi_{d}\right)^{n_{d}} .
$$

On the other hand it is clear that

$$
\left|\tilde{\chi}_{l}(z)\right|_{p}^{\prime},\left|\tilde{\chi}_{l}(z)^{-1}\right|_{p}^{\prime} \leq p^{C|\tilde{z}|_{\mathbb{Z}^{d}}}, \quad z \in A \times S
$$

where $|.|_{\mathbb{Z}^{d}}$ denotes the Euclidean norm on $\mathbb{Z}^{d}$. We have also

$$
\|\operatorname{Ad}(z)\|\left|\leq C\left\|\left|\operatorname{Ad}\left(\pi_{1}\right)\|\|^{n_{1}} \ldots\|\| \operatorname{Ad}\left(\pi_{d}\right) \|\right|^{n_{d}} \leq C p^{C|\tilde{z}|_{\mathbb{Z}^{d}}, \quad z \in A \times S .}\right.\right.
$$

Combining (20), (21), (22) we deduce that the triangular matrice $\mathscr{T}(z)$ that appears in (20) satisfies

$$
\left\||\mathscr{T}(z) \|| C \leq p^{C|\tilde{z}|_{\mathbb{Z}^{d}}}, \quad z \in A \times S .\right.
$$


Let now $z_{1}, z_{2}, \ldots, z_{k} \in A \times S$ and $1 \leq l \leq r$. We have

$$
\begin{aligned}
\left.\operatorname{Ad}\left(z_{1} z_{2} \ldots z_{k}\right)\right|_{W_{l}}= & \left.\operatorname{Ad}\left(\tilde{\tau}_{1} \tilde{\tau}_{2} \ldots \tilde{\tau}_{k}\right)\right|_{W_{l}} \circ \prod_{j=1}^{k}\left(\tilde{\chi}_{l}\left(z_{j}\right) I+\mathscr{T}\left(z_{j}\right)\right) \\
= & \left.\tilde{\chi}_{l}\left(z_{1} z_{2} \ldots z_{k}\right) \operatorname{Ad}\left(\tilde{\tau}_{1} \tilde{\tau}_{2} \ldots \tilde{\tau}_{k}\right)\right|_{W_{l}} \\
& \circ \sum_{\alpha, i_{j}}\left(\tilde{\chi}_{l}\left(z_{i_{1}}\right) \ldots \tilde{\chi}_{l}\left(z_{i_{\alpha}}\right)\right)^{-1} \mathscr{T}\left(z_{i_{1}}\right) \ldots \mathscr{T}\left(z_{i_{\alpha}}\right) .
\end{aligned}
$$

Using the fact that in the last sum all the terms corresponding to indexes $\alpha>n$ vanish and combining this with (21), (23) and the ultrametric property (19) we deduce that

$$
\|\left|A d\left(z_{1} z_{2} \ldots z_{k}\right)\right|_{W_{l}}|| \leq C\left|\tilde{\chi}_{l}\left(z_{1} z_{2} \ldots z_{k}\right)\right|_{p}^{\prime} p^{C \max _{1 \leq j \leq k}\left|\tilde{z}_{j}\right|_{\mathbb{Z}^{d}}}
$$

If we use the linear forms $L_{l}$ defined by (5) and (6) we then deduce

$$
\|\left|A d\left(z_{1} z_{2} \ldots z_{k}\right)\right|_{W_{l}}|| \leq C p^{\max _{1 \leq l \leq s} L_{l}\left(\tilde{z}_{1}+\ldots+\tilde{z}_{k}\right)+C \max _{1 \leq j \leq k}\left|\tilde{z}_{j}\right|_{\mathbb{Z}^{d}}}
$$

By the above considerations we have finally proved that

$$
\begin{gathered}
\left\|A d\left(z_{1} z_{2} \ldots z_{k}\right)\right\| \mid \leq C p^{\max _{1 \leq l \leq s} L_{l}\left(\zeta_{1}+\ldots+\zeta_{k}\right)+C \max _{1 \leq j \leq n}\left|\zeta_{j}\right|_{\mathbb{Z}^{d}},} \\
\zeta_{j}=p\left(z_{j}\right), \quad z_{j} \in A \times S, \quad j=1, \ldots, k, \quad k=1,2, \ldots
\end{gathered}
$$

If we apply this estimate to $u_{j}^{z_{1} z_{2} \ldots z_{j-1}}=\exp \left(\operatorname{Ad}\left(z_{1} \ldots z_{j-1}\right) v_{j}\right)$ where the $u_{j}$ 's, $v_{j}$ 's and $z_{j}$ 's are as in (16), (17), (18) we then deduce

$$
\begin{gathered}
\left\|A d\left(z_{1} \ldots z_{j-1}\right) v_{j}\right\| \leq C p^{\max _{1 \leq l \leq s} L_{l}\left(\zeta_{1}+\ldots+\zeta_{j-1}\right)+C \max _{1 \leq k \leq j-1}\left|\zeta_{k}\right|_{\mathbb{Z}^{d}}}, \\
\zeta_{j}=p\left(z_{j}\right), \quad j \geq 2 .
\end{gathered}
$$

Observe that the measure that controls the random walk $\left(S_{j}\right)_{j \in \mathrm{N}}$, defined by $S_{j}=\zeta_{0}+\zeta_{1}+\ldots+\zeta_{j}$ $\left(\zeta_{0}=0\right)$ is the symmetric measure $\check{\mu}$ defined by (8). The random variables $\zeta_{j}$ are in particular compactly supported and we have

$$
\left\|A d\left(z_{1} \ldots z_{j-1}\right) v_{j}\right\| \leq C p^{\max _{1 \leq l \leq r} L_{l}\left(\zeta_{1}+\ldots+\zeta_{j}\right)}, \quad \zeta_{j}=p\left(z_{j}\right), \quad j \geq 2 .
$$

Let us consider, for $n=1,2, \ldots$, the event $E_{n}$ defined by

$$
\begin{gathered}
E_{n}=\left(L_{l}\left(\zeta_{1}+\ldots+\zeta_{j}\right) \leq C, \quad j=1, \ldots, n, \quad l=1, \ldots, s\right. \\
\left.\left|\zeta_{1}+\ldots+\zeta_{n}\right|_{\mathbb{Z}^{d}} \leq C \sqrt{n}\right),
\end{gathered}
$$

where $C>0$ denotes an appropriate large constant. Using (15), Campbell-Hausdorff, (24) and the ultrametric property (19) we see that the event $E_{n}$ verifies

$$
E_{n} \subset\left[X_{n} \in B_{n}\right]
$$


where $B_{n}$ is defined by

$$
B_{n}=\exp (\{u \in \mathscr{U}, \quad\|u\| \leq C\}) .\left\{z \in A \times S, \quad|p(z)|_{\mathbb{Z}^{d}} \leq A n^{1 / 2}\right\} .
$$

It is easy to see that $d^{r} g$ shows that

$$
\left|B_{n}\right| \leq C n^{\frac{d}{2}}, \quad n=1,2, \ldots
$$

It remains to estimate the probability of the event (25). Let $\Pi$ denote the polyhedral region in $\mathbb{Z}^{d}$ defined by

$$
\Pi=\left\{z \in \mathbb{Z}^{d}, \quad L_{j}(z) \leq C, \quad j=1, \ldots, s\right\},
$$

where $C$ denotes the same constant as in (25). Let $h_{n}(x, y), n=1,2, \ldots, x, y \in \Pi$, denote the transition kernel corresponding to the random walk $\left(S_{j}\right)_{j \in \mathbf{N}}$ with killing outside of $\Pi$. Precise lower estimates for the kernel $h_{n}(x, y)$ can be obtained thanks to the results of [26] and [27]. To write down these estimates we need the following notations. Let us assume that $d \geq 2$ and let $0<u_{0}(\sigma)$, $\sigma \in \Pi_{\Sigma}$, denote the eigenfunction corresponding to the first Dirichlet dirichlet eigenvalue of the region $\Pi_{\Sigma}$ defined by (10). Let $u(x)$ be the function defined on $\bar{\Pi}_{\mathscr{L}}$ by

$$
u(x)=u(r, \sigma)=r^{a} u_{0}(\sigma), \quad x=(r, \sigma) \in \mathbb{R}_{+}^{*} \times \Pi_{\Sigma}
$$

where

$$
a=\frac{\sqrt{(d-2)^{2}+4 \lambda}-(d-2)}{2},
$$

and where $(r, \sigma)$ denote the polar coordinates on $\mathbb{R}_{+}^{*} \times \Sigma$. The function $u$ defined in this way is a positive function harmonic in $\Pi_{\mathscr{L}}$ which vanishes on $\partial \Pi_{\mathscr{L}}$ (cf. [24]). In the case where $d=1$, the function $u$ is defined by $u(x) \equiv x, x \in \mathbb{R}_{+}^{*}$.

It follows easily from [27] (cf. estimate (2) p. 359) and [26] (cf. Theorem 1 and estimate (0.3.4)) that there exists $C>0$ such that

$$
h_{n}(0, y) \geq \frac{u(-y)}{C n^{a+d / 2}}, \quad|y| \leq C \sqrt{n}, \quad n \geq C .
$$

We have therefore

$$
\mathbb{P}\left(E_{n}\right) \geq \frac{1}{C n^{a+d / 2}} \sum_{y \in \Pi,|y| \leq C \sqrt{n}} u(-y) .
$$

Using the homogeneity of $u$ we deduce then that

$$
\mathbb{P}\left(E_{n}\right) \geq \frac{1}{C n^{a+d / 2}} \int_{0}^{C \sqrt{n}} r^{a+d-1} d r=\frac{1}{C n^{a / 2}} .
$$

The lower estimate (11) is an immediate consequence of (13), (26), (27) and (28). This completes the proof of Theorem 1 . 


\section{Metabelian $p$-adic groups}

Our aim in in this section is to show that in the case of metabelian groups the lower estimate (11) can be complemented with a similar upper bound. We keep the notation of $\S 2$. We shall denote by $\mathbb{Z}_{p}^{*}=\left\{x \in \mathbb{Q}_{p}^{*}, \quad|x|_{p}=1\right\}$ and by $\mathbb{Z}_{p}=\left\{x \in \mathbb{Q}_{p},|x|_{p} \leq 1\right\}$. Let $d x$ denote the Haar measure on $\mathbb{Q}_{p}$ normalized by $d x\left(\mathbb{Z}_{p}\right)=1$ and let $d^{*} x$ denote the Haar measure on $\mathbb{Q}_{p}^{*}$ normalized by $d^{*} x\left(\mathbb{Z}_{p}^{*}\right)=1$. Let us fix $k, l \geq 2$ and consider

$$
G=\mathbb{Q}_{p}^{k} \ltimes_{\sigma}\left(\mathbb{Q}_{p}^{*}\right)^{l}
$$

the semi-direct product of $\left(\mathbb{Q}_{p}^{*}\right)^{l}$ with the vector space $\mathbb{Q}_{p}^{k}$ where $\left(\mathbb{Q}_{p}^{*}\right)^{l}$ acts on $\mathbb{Q}_{p}^{k}$ by

$$
x=\left(x_{1}, \ldots, x_{k}\right) \longrightarrow \sigma(y) x=\left(\chi_{1}(y) x_{1}, \ldots, \chi_{k}(y) x_{k}\right), \quad y \in\left(\mathbb{Q}_{p}^{*}\right)^{l},
$$

where $\chi_{1}, \ldots, \chi_{k}$ denote $k$ morphisms

$$
\chi_{1}, \ldots, \chi_{k}:\left(\mathbb{Q}_{p}^{*}\right)^{l} \longrightarrow \mathbb{Q}_{p}^{*} .
$$

More precisely, we assume that the multiplication in $G$ is given by

$$
\begin{gathered}
g \cdot g^{\prime}=(x ; y) \cdot\left(x^{\prime} ; y^{\prime}\right)=\left(x+\sigma(y) x^{\prime} ; y \cdot y^{\prime}\right) \\
=\left(x_{1}+\chi_{1}(y) x_{1}^{\prime}, x_{2}+\chi_{2}(y) x_{2}^{\prime}, \ldots, x_{k}+\chi_{k}(y) x_{k}^{\prime} ; y_{1} \cdot y_{1}^{\prime}, y_{2} \cdot y_{2}^{\prime}, \ldots, y_{l} \cdot y_{l}^{\prime}\right) \\
g=(x, y), \quad g^{\prime}=\left(x^{\prime}, y^{\prime}\right) \in G ; \quad x=\left(x_{1}, \ldots, x_{k}\right), x^{\prime}=\left(x_{1}^{\prime}, \ldots, x_{k}^{\prime}\right) \in \mathbb{Q}_{p}^{k} ; \\
y=\left(y_{1}, \ldots, y_{l}\right), \quad y=\left(y_{1}^{\prime}, \ldots, y_{l}^{\prime}\right) \in\left(\mathbb{Q}_{p}^{*}\right)^{l} .
\end{gathered}
$$

We shall denote by

$$
d^{r} g=d x d^{*} y=d x_{1} \ldots d x_{k} d^{*} y_{1} \ldots d^{*} y_{l} ; \quad d^{l} g=d g=\delta(g)^{-1} d^{r} g
$$

the right and the left invariant Haar measure on $G$. The modular function $\delta(g)$ is given by

$$
\delta(g)=\delta(y)=\left|\chi_{1}(y)\right| \ldots\left|\chi_{k}(y)\right|, \quad g=(x, y) \in G
$$

Let $d \mu(g)=\varphi(g) d^{r}(g)$ denote the symmetric, compactly supported, probability measure on $G$ defined by

$$
\begin{gathered}
\varphi(g)=\alpha \delta^{-1 / 2}(g) I_{\mathbb{Z}_{p}}\left(x_{1}\right) \ldots I_{\mathbb{Z}_{p}}\left(x_{k}\right) I_{\mathbb{Z}_{p}}\left(\chi_{1}(y)^{-1} x_{1}\right) \ldots I_{\mathbb{Z}_{p}}\left(\chi_{k}(y)^{-1} x_{k}\right) \\
\times I_{\left(p^{-1} \mathbb{Z}_{p}^{*} \cup \mathbb{Z}_{p}^{*} \cup p \mathbb{Z}_{p}^{*}\right)}\left(y_{1}\right) \ldots I_{\left(p^{-1} \mathbb{Z}_{p}^{*} \cup \mathbb{Z}_{p}^{*} \cup p \mathbb{Z}_{p}^{*}\right)}\left(y_{l}\right), \\
g=\left(x_{1}, \ldots, x_{k} ; y_{1}, \ldots, y_{l}\right) \in G .
\end{gathered}
$$

The notation $I_{A}$ is used here to denote the indicator of a subset $A$ and $\alpha>0$ denotes an appropriate positive constant. 
We shall assume that the $\chi_{j}$ 's in (30) verify the condition

$$
\left|\chi_{j}\right| \not \equiv 1, \quad j=1, \ldots, k .
$$

This garantees (cf. §2) that the group $G$ defined by (29) is compactly generated and it is easy to see that $\operatorname{supp}(\mu)$ is a generating set of the group $G$. We shall denote, as in $\S 2$, by $L_{j}, j=1, \ldots, k$, the $k$ linear forms on $\mathbb{Z}^{l}$ induced by the $\chi_{j}$ 's and we shall assume that these linear forms induce a "Weyl chamber" $\Pi=\left\{x \in \mathbb{R}^{l}, \quad \tilde{L}_{j}(x)>0, \quad j=1,2, \ldots, k\right\}$ as in $\S 2$. We shall denote by $\Sigma=\left\{x \in \mathbb{R}^{l},|x|=\right.$ $1\}$ the unit sphere in $\mathbb{R}^{l}$, by $\Pi_{\Sigma}=\Sigma \cap \Pi$ and by $\lambda$ be the first Dirichlet eigenvalue of the region $\Pi_{\Sigma}$. We have then the following:

Theorem 2. Let $G=\mathbb{Q}_{p}^{k} \ltimes_{\sigma}\left(\mathbb{Q}_{p}^{*}\right)^{l}$ and let $\mu \in \mathbf{P}(G)$ be as above. Let $d \mu^{* n}(g)=d(\mu * \ldots * \mu)(g)=$ $\varphi_{n}(g) d^{r} g$ denote the $n^{\text {th }}$ convolution power of $\mu$. Then there exists $C>0$ such that

$$
\varphi_{n}(e) \leq \frac{C}{n^{1+\frac{\sqrt{(l-2)^{2}+4 \lambda}}{2}}}, \quad n=1,2, \ldots
$$

The first step to prove Theorem 2 consists in establishing an explicit formula for $\varphi_{n}(g), g \in G$.

In what follows we shall use the notation $x_{i}=\left(x_{i, 1}, \ldots, x_{i, k}\right)\left(\operatorname{resp} y_{i}=\left(y_{i, 1}, \ldots, y_{i, l}\right)\right)$ for $x_{i} \in \mathbb{Q}_{p}^{k}$, $i=1,2, \ldots$ (resp. $y_{i} \in\left(\mathbb{Q}_{p}^{*}\right)^{l}, i=1,2, \ldots$ ). Let us fix $g=(\xi, \zeta)=\left(\xi_{1}, \ldots, \xi_{k} ; \zeta_{1}, \ldots, \zeta_{l}\right) \in G$ and $n=1,2, \ldots$. By definition of convolution product we have:

$$
\begin{aligned}
& \varphi_{n+1}(g)=\int_{G} \varphi_{n}(h) \varphi_{1}\left(h^{-1} g\right) d h \\
&=\int_{G} \varphi_{1}\left(h^{-1} g\right) \delta\left(h^{-1}\right) \varphi_{n}(h) d^{r} h \\
&=\int_{G} \varphi_{1}\left(h^{-1} g\right) \delta\left(h^{-1}\right) d \mu^{* n}(h) \\
&=\int_{G} \ldots \int_{G} \varphi_{1}\left(\left(g_{1} \ldots g_{n}\right)^{-1} g\right) \delta\left(g_{1} \ldots g_{n}\right)^{-1} d \mu\left(g_{1}\right) \ldots d \mu\left(g_{n}\right) \\
&=\int_{G} \ldots \int_{G} \varphi_{1}\left(-\left(\sigma\left(y_{1} \ldots y_{n}\right)^{-1} x_{1}+\sigma\left(y_{2} \ldots y_{n}\right)^{-1} x_{2}+\ldots+\sigma\left(y_{n}\right)^{-1} x_{n}\right)\right. \\
&\left.+\sigma\left(y_{1} \ldots y_{n}\right)^{-1} \xi,\left(y_{1} \ldots y_{n}\right)^{-1} \zeta\right) \varphi_{1}\left(x_{1}, y_{1}\right) \ldots \varphi\left(x_{n}, y_{n}\right) \\
& \delta\left(y_{1} \ldots y_{n}\right)^{-1} d x_{1} d^{*} y_{1} \ldots d x_{n} d^{*} y_{n} .
\end{aligned}
$$


An obvious change of variables combined with Fubini gives

$$
\begin{aligned}
\varphi_{n+1}(g)=\int_{\left(\mathbb{Q}_{p}^{*}\right)^{l}} \ldots \int_{\left(\mathbb{Q}_{p}^{*}\right)^{l}}\left[\int _ { \mathbb { Q } _ { p } ^ { k } } \ldots \int _ { \mathbb { Q } _ { p } ^ { k } } \left[\varphi _ { 1 } \left(-\left(x_{1}+x_{2}+\ldots .+x_{n}\right)\right.\right.\right. \\
\left.+\sigma\left(y_{1} \ldots y_{n}\right)^{-1} \xi,\left(y_{1} \ldots y_{n}\right)^{-1} \zeta\right) \varphi_{1}\left(\sigma\left(y_{1} \ldots y_{n}\right) x_{1}, y_{1}\right) \\
\left.\left.\varphi_{1}\left(\sigma\left(y_{2} \ldots y_{n}\right) x_{2}, y_{2}\right) \ldots \varphi_{1}\left(\sigma\left(y_{n}\right) x_{n}, y_{n}\right)\right] d x_{1} \ldots d x_{n}\right] \\
\prod_{i=2}^{n}\left|\chi_{1}\left(y_{i} \ldots y_{n}\right)\right| \ldots\left|\chi_{k}\left(y_{i} \ldots y_{n}\right)\right| d^{*} y_{1} \ldots d^{*} y_{n}
\end{aligned}
$$

where we used (31). Let us consider the product

$$
\prod_{i=1}^{n} \varphi_{1}\left(\sigma\left(y_{i} \ldots y_{n}\right) x_{i}, y_{i}\right)
$$

that appears in equation (33). By (32), this product is equal to

$$
\begin{aligned}
& \alpha^{n} \prod_{i=1}^{n} \delta^{-1 / 2}\left(y_{i}\right) \prod_{i=1}^{n}\left(I_{\mathbb{Z}_{p}}\left(\chi_{1}\left(y_{i} \ldots y_{n}\right) x_{i, 1}\right) \ldots I_{\mathbb{Z}_{p}}\left(\chi_{k}\left(y_{i} \ldots y_{n}\right) x_{i, k}\right)\right) \\
& \times \prod_{i=1}^{n-1}\left(I_{\mathbb{Z}_{p}}\left(\chi_{1}\left(y_{i+1} \ldots y_{n}\right) x_{i, 1}\right) \ldots I_{\mathbb{Z}_{p}}\left(\chi_{k}\left(y_{i+1} \ldots y_{n}\right) x_{i, k}\right)\right) \\
& \times I_{\mathbb{Z}_{p}}\left(x_{n, 1}\right) \ldots I_{\mathbb{Z}_{p}}\left(x_{n, k}\right) \prod_{i=1}^{n} I_{\left(p^{-1} \mathbb{Z}_{p}^{*} \cup \mathbb{Z}_{p}^{*} \cup p \mathbb{Z}_{p}^{*}\right)}\left(y_{i, 1}\right) \ldots I_{\left(p^{-1} \mathbb{Z}_{p}^{*} \cup \mathbb{Z}_{p}^{*} \cup p \mathbb{Z}_{p}^{*}\right)}\left(y_{i, l}\right) \\
& =\alpha^{n} \prod_{i=1}^{n} \delta^{-1 / 2}\left(y_{i}\right) \prod_{i=1}^{n-1}\left(I_{\left|\chi_{1}\left(y_{i+1} \ldots y_{n}\right)\right| \max \left(1,\left|\chi_{1}\left(y_{i}\right)\right|\right) \mathbb{Z}_{p}}\left(x_{i, 1}\right)\right. \\
& \left.\ldots I_{\left|\chi_{k}\left(y_{i+1} \ldots y_{n}\right)\right| \max \left(1,\left|\chi_{k}\left(y_{i}\right)\right|\right) \mathbb{Z}_{p}}\left(x_{i, k}\right)\right) \\
& I_{\max \left(1,\left|\chi_{1}\left(y_{n}\right)\right|\right) \mathbb{Z}_{p}}\left(x_{n, 1}\right) \ldots I_{\max \left(1,\left|\chi_{k}\left(y_{n}\right)\right|\right) \mathbb{Z}_{p}}\left(x_{n, k}\right) \\
& \prod_{i=1}^{n} I_{\left(p^{-1} \mathbb{Z}_{p}^{*} \cup \mathbb{Z}_{p}^{*} \cup p \mathbb{Z}_{p}^{*}\right)}\left(y_{i, 1}\right) \ldots I_{\left(p^{-1} \mathbb{Z}_{p}^{*} \cup \mathbb{Z}_{p}^{*} \cup p \mathbb{Z}_{p}^{*}\right)}\left(y_{i, l}\right) .
\end{aligned}
$$

We also have

$$
\begin{aligned}
\varphi_{1}( & \left.-\left(x_{1}+x_{2}+\ldots .+x_{n}\right)+\sigma\left(y_{1} \ldots y_{n}\right)^{-1} \xi,\left(y_{1} \ldots y_{n}\right)^{-1} \zeta\right) \\
= & \alpha \delta^{-1 / 2}\left(\left(y_{1} \ldots y_{n}\right)^{-1} \zeta\right) \\
& \times I_{\max \left(1,\left|\chi_{1}\left(y_{1} \ldots y_{n}\right) \zeta^{-1}\right|\right) \mathbb{Z}_{p}}\left(x_{1,1}+x_{2,1}+\ldots .+x_{n, 1}-\chi_{1}\left(y_{1} \ldots y_{n}\right)^{-1} \xi_{1}\right) \\
& \ldots I_{\max \left(1,\left|\chi_{k}\left(y_{1} \ldots y_{n}\right)^{-1} \zeta^{-1}\right|\right) \mathbb{Z}_{p}}\left(x_{1, k}+x_{2, k}+\ldots .+x_{n, k}-\chi_{k}\left(y_{1} \ldots y_{n}\right)^{-1} \xi_{k}\right) \\
& \times I_{\left(p^{-1} \mathbb{Z}_{p}^{*} \cup \mathbb{Z}_{p}^{*} \cup p \mathbb{Z}_{p}^{*}\right)}\left(\left(y_{1,1} \ldots y_{n, 1}\right)^{-1} \zeta_{1}\right) \ldots I_{\left(p^{-1} \mathbb{Z}_{p}^{*} \cup \mathbb{Z}_{p}^{*} \cup p \mathbb{Z}_{p}^{*}\right)}\left(\left(y_{1, l} \ldots y_{n, l}\right)^{-1} \zeta_{l}\right) .
\end{aligned}
$$


On the other hand we shall set

$$
\begin{gathered}
m(y)=\alpha \delta^{-1 / 2}(y) \prod_{j=1}^{k} \min \left(1,\left|\chi_{j}(y)\right|\right) I_{\left(p^{-1} \mathbb{Z}_{p}^{*} \cup \mathbb{Z}_{p}^{*} \cup p \mathbb{Z}_{p}^{*}\right)}\left(y_{1}\right) \ldots I_{\left(p^{-1} \mathbb{Z}_{p}^{*} \cup \mathbb{Z}_{p}^{*} \cup p \mathbb{Z}_{p}^{*}\right)}\left(y_{l}\right), \\
y=\left(y_{1}, \ldots, y_{l}\right) \in\left(\mathbb{Q}_{p}^{*}\right)^{l} .
\end{gathered}
$$

What motivates this notation is the fact that

$$
\int_{\mathbb{Q}_{p}^{k}} \varphi(x, y) d x=m(y), \quad y \in\left(\mathbb{Q}_{p}^{*}\right)^{l} .
$$

Setting

$$
\begin{aligned}
& A\left(y_{1}, y_{2}, \ldots, y_{n}\right)=m\left(\left(y_{1} \ldots y_{n}\right)^{-1} \zeta\right) \prod_{j=1}^{k} \max \left(1,\left|\chi_{j}\left(y_{1} \ldots y_{n}\right) \zeta^{-1}\right|\right) \\
& \times \prod_{i=1}^{n} \max \left(1,\left|\chi_{1}\left(y_{i}\right)\right|^{-1}\right) \ldots \max \left(1,\left|\chi_{k}\left(y_{i}\right)\right|^{-1}\right) m\left(y_{i}\right) \text {, } \\
& B_{j}\left(x_{1}, x_{2}, \ldots, x_{n} ; y_{1}, y_{2}, \ldots, y_{n}\right)=B_{j}\left(x_{1}, x_{2}, \ldots, x_{n}\right) \\
& =I_{\max \left(1,\left|\chi_{j}\left(y_{1} \ldots y_{n}\right) \zeta^{-1}\right|\right) \mathbb{Z}_{p}}\left(x_{1, j}+x_{2, j}+\ldots\right. \\
& \left.+x_{n, j}-\chi_{j}\left(y_{1} \ldots y_{n}\right)^{-1} \xi_{j}\right) I_{\max \left(1,\left|\chi_{j}\left(y_{n}\right)\right|\right) \mathbb{Z}_{p}}\left(x_{n, j}\right) \\
& \times \prod_{i=1}^{n-1} I_{\left|\chi_{j}\left(y_{i+1} \ldots y_{n}\right)\right| \max \left(1,\left|\chi_{j}\left(y_{i}\right)\right|\right) \mathbb{Z}_{p}}\left(x_{i, j}\right), \\
& j=1,2, \ldots k \text {, }
\end{aligned}
$$

we rewrite (33) as

$$
\begin{gathered}
\varphi_{n+1}(g)=\int_{\left(\mathbb{Q}_{p}^{*}\right)^{l}} \ldots \int_{\left(\mathbb{Q}_{p}^{*}\right)^{l}}\left[\int_{\mathbb{Q}_{p}^{k}} \ldots \int_{\mathbb{Q}_{p}^{k}} \prod_{j=1}^{k} B_{j}\left(x_{1}, x_{2}, \ldots, x_{n}\right) d x_{1} \ldots d x_{n}\right] \\
A\left(y_{1}, y_{2}, \ldots, y_{n}\right) \prod_{i=2}^{n}\left|\chi_{1}\left(y_{i} \ldots y_{n}\right)\right| \ldots\left|\chi_{k}\left(y_{i} \ldots y_{n}\right)\right| \\
d^{*} y_{1} \ldots d^{*} y_{n} .
\end{gathered}
$$

The next step is to calculate

$$
\int_{\mathbb{Q}_{p}} \ldots \int_{\mathbb{Q}_{p}} B_{j}\left(x_{1}, x_{2}, \ldots, x_{n}\right) d x_{1, j} d x_{2, j} \ldots d x_{n, j}, \quad j=1, \ldots, k .
$$


Towards this we observe that:

$$
\begin{gathered}
\int_{\mathbb{Q}_{p}} \ldots \int_{\mathbb{Q}_{p}} I_{\max \left(1,\left|\chi_{j}\left(y_{1} \ldots y_{n}\right) \zeta^{-1}\right|\right) \mathbb{Z}_{p}}\left(x_{1, j}+\ldots+x_{n, j}-\chi_{j}\left(y_{1} \ldots y_{n}\right)^{-1} \xi_{j}\right) \\
I_{\max \left(1,\left|\chi_{j}\left(y_{n}\right)\right|\right) \mathbb{Z}_{p}}\left(x_{n, j}\right) \prod_{i=1}^{n-1} I_{\left|\chi_{j}\left(y_{i+1} \ldots y_{n}\right)\right| \max \left(1,\left|\chi_{j}\left(y_{i}\right)\right|\right) \mathbb{Z}_{p}}\left(x_{i, j}\right) d x_{1, j} d x_{2, j} \ldots d x_{n, j} \\
=\int_{\mathbb{Q}_{p}}\left(I_{\left|\chi_{j}\left(y_{2} \ldots y_{n}\right)\right| \max \left(1,\left|\chi_{j}\left(y_{1}\right)\right|\right) \mathbb{Z}_{p}} * \cdots * I_{\left|\chi_{j}\left(y_{n}\right)\right| \max \left(1,\left|\chi_{j}\left(y_{n-1}\right)\right|\right) \mathbb{Z}_{p}} * I_{\max \left(1,\left|\chi_{j}\left(y_{n}\right)\right|\right) \mathbb{Z}_{p}}\right)(x) \\
I_{\max \left(1,\left|\chi_{j}\left(y_{1} \ldots y_{n}\right) \zeta^{-1}\right|\right) \mathbb{Z}_{p}}\left(x-\chi_{j}\left(y_{1} \ldots y_{n}\right)^{-1} \xi_{j}\right) d x
\end{gathered}
$$

where $*$ denotes the usual convolution in $\mathbb{Q}_{p}$ (cf. [21]). Taking Fourier transform (cf. [21]) we obtain

$$
\begin{aligned}
& \int_{\mathbb{Q}_{p}} \ldots \int_{\mathbb{Q}_{p}} B_{j}\left(x_{1}, x_{2}, \ldots, x_{n}\right) d x_{1, j} d x_{2, j} \ldots d x_{n, j} \\
& =\operatorname{Min}\left[\min _{1 \leq i \leq n-1}\left(\left|\chi_{j}\left(y_{i+1} \ldots y_{n}\right)\right| \max \left(1,\left|\chi_{j}\left(y_{i}\right)\right|\right)\right) ; \max \left(1,\left|\chi_{j}\left(y_{n}\right)\right|\right)\right] \\
& \quad \min \left(1,\left|\chi_{j}\left(y_{n}\right)\right|^{-1}\right) \prod_{i=1}^{n-1}\left|\chi_{j}\left(y_{i+1} \ldots y_{n}\right)\right|^{-1} \min \left(1,\left|\chi_{j}\left(y_{i}\right)\right|^{-1}\right) \\
& \quad \times\left(I_{\operatorname{Min}\left[\min _{1 \leq i \leq n-1}\left(\left|\chi_{j}\left(y_{i+1} \ldots y_{n}\right)\right| \max \left(1,\left|\chi_{j}\left(y_{i}\right)\right|\right)\right) ; \max \left(1,\left|\chi_{j}\left(y_{i}\right)\right|\right)\right] \mathbb{Z}_{p}} *\right. \\
& \left.\quad I_{\max \left(1,\left|\chi_{j}\left(y_{1} \ldots y_{n}\right) \zeta\right|^{-1}\right) \mathbb{Z}_{p}}\right)\left(\chi_{j}\left(y_{1} \ldots y_{n}\right)^{-1} \xi_{j}\right) .
\end{aligned}
$$

Let us denote by $S_{j}^{\zeta}\left(y_{1}, \ldots, y_{n}\right) \subset \mathbb{Q}_{p}, y_{1}, \ldots, y_{n} \in \mathbb{Q}_{p}^{*}$ the subset defined by

$$
\begin{gathered}
S_{j}^{\zeta}\left(y_{1}, \ldots, y_{n}\right)=\operatorname{Min}\left[\min _{1 \leq i \leq n-1}\left(\left|\chi_{j}\left(y_{i+1} \ldots y_{n}\right)\right| \max \left(1,\left|\chi_{j}\left(y_{i}\right)\right|\right)\right) ;\right. \\
\left.\max \left(1,\left|\chi_{j}\left(y_{n}\right)\right|\right)\right] \wedge \max \left(1,\left|\chi_{j}\left(y_{1} \ldots y_{n}\right) \zeta^{-1}\right|\right) \mathbb{Z}_{p} .
\end{gathered}
$$

With this notation we have

$$
\begin{aligned}
\int_{\mathbb{Q}_{p}} & \ldots \int_{\mathbb{Q}_{p}} B_{j}\left(x_{1}, x_{2}, \ldots, x_{n}\right) d x_{1, j} d x_{2, j} \ldots d x_{n, j} \\
= & \min \left(1 ; \operatorname{Min}\left(\min _{1 \leq i \leq n-1}\left(\left|\chi_{j}\left(y_{i+1} \ldots y_{n}\right)\right| \max \left(1,\left|\chi_{j}\left(y_{i}\right)\right|\right)\right)\right.\right. \\
& \left.\left.\max \left(1,\left|\chi_{j}\left(y_{n}\right)\right|\right)\right) \min \left(1,\left|\chi_{j}\left(y_{1} \ldots y_{n}\right)^{-1} \zeta\right|\right)\right) \min \left(1,\left|\chi_{j}\left(y_{n}\right)\right|^{-1}\right) \\
& \prod_{i=1}^{n-1}\left|\chi_{j}\left(y_{i+1} \ldots y_{n}\right)\right|^{-1} \min \left(1,\left|\chi_{j}\left(y_{i}\right)\right|^{-1}\right) I_{S_{j}^{\zeta}\left(y_{1}, \ldots, y_{n}\right)}\left(\chi_{j}\left(y_{1} \ldots y_{n}\right)^{-1} \xi_{j}\right),
\end{aligned}
$$




$$
j=1, \ldots, k .
$$

Putting together (33), (36), (37), (38) and (39) and taking into account the fact that

$$
\begin{gathered}
\prod_{j=1}^{k} \min \left(1,\left|\chi_{j}\left(y_{n}\right)\right|^{-1}\right) \prod_{i=1}^{n-1}\left(\prod_{j=1}^{k} \min \left(1,\left|\chi_{j}\left(y_{i}\right)\right|^{-1}\right)\right) \\
\times \prod_{i=1}^{n} \max \left(1,\left|\chi_{1}\left(y_{i}\right)\right|^{-1}\right) \ldots \max \left(1,\left|\chi_{k}\left(y_{i}\right)\right|^{-1}\right) \\
=\prod_{j=1}^{k}\left|\chi_{j}\left(y_{1} \ldots y_{n}\right)\right|^{-1}=\delta^{-1}\left(y_{1} \ldots y_{n}\right),
\end{gathered}
$$

and that

$$
\begin{gathered}
\delta^{-1}\left(y_{1} \ldots y_{n}\right) \prod_{j=1}^{k} \max \left(1,\left|\chi_{j}\left(y_{1} \ldots y_{n}\right) \zeta^{-1}\right|\right) m\left(\left(y_{1} \ldots y_{n}\right)^{-1} \zeta\right) \\
=\alpha \delta^{-1 / 2}\left(y_{1} \ldots y_{n}\right) \delta^{-1 / 2}(\zeta) I_{\left(p^{-1} \mathbb{Z}_{p}^{*} \cup \mathbb{Z}_{p}^{*} \cup p \mathbb{Z}_{p}^{*}\right)}\left(\left(y_{1,1} \ldots y_{n, 1}\right)^{-1} \zeta_{1}\right) \\
\ldots I_{\left(p^{-1} \mathbb{Z}_{p}^{*} \cup \mathbb{Z}_{p}^{*} \cup p \mathbb{Z}_{p}^{*}\right)}\left(\left(y_{1, l} \ldots y_{n, l}\right)^{-1} \zeta_{l}\right),
\end{gathered}
$$

we finally deduce the formula

$$
\begin{aligned}
(40) \varphi_{n+1}(g)=\alpha \delta^{-1 / 2}(\zeta) & \int_{\left(\mathbb{Q}_{p}^{*}\right)^{l}} \ldots \int_{\left(\mathbb{Q}_{p}^{*}\right)} \prod_{j=1}^{k} \operatorname{Min}(1 ; \\
& \min \left(\min _{1 \leq i \leq n-1}\left(\left|\chi_{j}\left(y_{i+1} \ldots y_{n}\right)\right| \max \left(1,\left|\chi_{j}\left(y_{i}\right)\right|\right)\right) ;\right. \\
& \left.\left.\max \left(1,\left|\chi_{j}\left(y_{n}\right)\right|\right)\right) \min \left(1,\left|\chi_{j}\left(y_{1} \ldots y_{n}\right)^{-1} \zeta\right|\right)\right) \\
& \delta^{-1 / 2}\left(y_{1} \ldots y_{n}\right) \prod_{j=1}^{k} I_{S_{j}^{\zeta}\left(y_{1}, \ldots, y_{n}\right)}\left(\chi_{j}\left(y_{1} \ldots y_{n}\right)^{-1} \xi_{j}\right) \\
& \prod_{j=1}^{l} I_{\left(p^{-1} \mathbb{Z}_{p}^{*} \mathbb{Z}_{p}^{*} \cup p \mathbb{Z}_{p}^{*}\right)}\left(\left(y_{1, j} \ldots y_{n, j}\right)^{-1} \zeta_{l}\right) \\
& m\left(y_{1}\right) \ldots m\left(y_{n}\right) d^{*} y_{1} \ldots d^{*} y_{n} .
\end{aligned}
$$

The next step is to give a probabilistic interpretation of (40). Towards that let us consider a sequence of independent identically $\left(\mathbb{Q}_{p}^{*}\right)^{l}$-valued random variables $Y_{1}, Y_{2}, \ldots$ with distribution on $\left(\mathbb{Q}_{p}^{*}\right)^{l}$ given by

$$
\mathbb{P}\left[Y_{j} \in d y\right]=m(y) d^{*} y
$$


where $m(y)$ is as in (34). Observe that by (35) we have $\int m(y) d^{*} y=1$. The formula (40) can be rewritten

$$
\begin{aligned}
\varphi_{n+1}(g)=\alpha \delta^{-1 / 2}(\zeta) \mathbb{E} & {\left[\prod _ { j = 1 } ^ { k } \operatorname { M i n } \left(1 ; \min \left(\min _{1 \leq i \leq n-1}\left(\left|\chi_{j}\left(Y_{i+1} \ldots Y_{n}\right)\right| \max \left(1,\left|\chi_{j}\left(Y_{i}\right)\right|\right)\right) ;\right.\right.\right.} \\
& \left.\left.\max \left(1,\left|\chi_{j}\left(Y_{n}\right)\right|\right)\right) \min \left(1,\left|\chi_{j}\left(Y_{1} \ldots Y_{n}\right)^{-1} \zeta\right|\right)\right) \\
& \delta^{-1 / 2}\left(Y_{1} \ldots Y_{n}\right) \prod_{j=1}^{k} I_{S_{j}^{\zeta}\left(Y_{1}, \ldots, Y_{n}\right)}\left(\chi_{j}\left(Y_{1} \ldots Y_{n}\right)^{-1} \xi_{j}\right) \\
& \left.\prod_{j=1}^{l} I_{\left(p^{-1} \mathbb{Z}_{p}^{*} \cup \mathbb{Z}_{p}^{*} \cup p \mathbb{Z}_{p}^{*}\right)}\left(\left(Y_{1, j} \ldots Y_{n, j}\right)^{-1} \zeta_{j}\right)\right] .
\end{aligned}
$$

In the case $g=e$ the above formula simplifies considerably and we obtain

$$
\begin{aligned}
& \varphi_{n+1}(e) \leq C \mathbb{E}\left(\prod_{j=1}^{k} \min \left[1, \min _{1 \leq i \leq n}\left|\chi_{j}\left(Y_{i} \ldots Y_{n}\right)\right|\right]\right. \\
& \times \prod_{j=1}^{l} I\left(p^{-1} \mathbb{Z}_{p}^{*} \cup \mathbb{Z}_{p}^{*} \cup p \mathbb{Z}_{p}^{*}\right) \\
&\left.\left(\left(Y_{1, j} \ldots Y_{n, j}\right)^{-1}\right)\right) .
\end{aligned}
$$

Now we project the random walk $Y_{1} \ldots Y_{n}$ controlled by (41) on $\mathbb{Z}^{l} \subset \mathbb{R}^{l}$ and we assume that $\mathbb{R}^{l}$ is equipped with the Euclidean structure ensuring (9). We shall denote by $S_{n}=X_{1}+X_{2}+\ldots+X_{n}$ $(n \geq 1)$ the random walk obtained via this projection. We have

$$
\varphi_{n+1}(e) \leq C \mathbb{E}\left(\prod_{j=1}^{k} \min _{1 \leq i \leq n} p^{L_{j}\left(S_{n}-S_{i}\right)}, \quad S_{n} \in K\right), \quad n=1,2, \ldots
$$

where $K$ denotes a neighbourhood of the origin in $\mathbb{Z}^{l}$. It follows then that

$$
\varphi_{n+1}(e) \leq C \mathbb{E}\left(\prod_{j=1}^{k} p^{-\max _{1 \leq i \leq n} L_{j}\left(S_{i}\right)}, \quad S_{n} \in K\right), \quad n=1,2, \ldots
$$

and therefore

$$
\begin{gathered}
\varphi_{n+1}(e) \leq C \sum_{\lambda_{1} \geq 0, \ldots, \lambda_{k} \geq 0} p^{-\sum_{i=1}^{k} \lambda_{i} \mathbb{P}\left(\max _{0 \leq i \leq n} L_{1}\left(S_{i}\right)=\lambda_{1}, \ldots,\right.} \\
\left.\max _{0 \leq i \leq n} L_{k}\left(S_{i}\right)=\lambda_{k} ; \quad S_{n} \in K\right), \quad n=1,2, \ldots
\end{gathered}
$$

Let us now fix $x_{0} \in \Pi$ such that

$$
L_{j}\left(x_{0}\right)>0, \quad j=1, \ldots, k
$$

Let

$$
\lambda_{0}=\min _{1 \leq j \leq k} L_{j}\left(x_{0}\right)
$$


We have

$$
L_{j}\left(u_{0}\right)=L_{j}\left(x_{0} / \lambda_{0}\right) \geq 1
$$

It follows then form (42) that

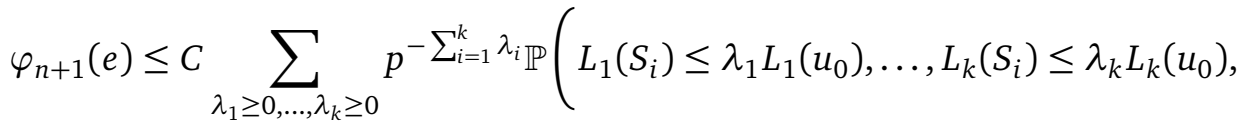

$$
\begin{aligned}
& \left.0 \leq i \leq n ; \quad S_{n} \in K\right)
\end{aligned}
$$

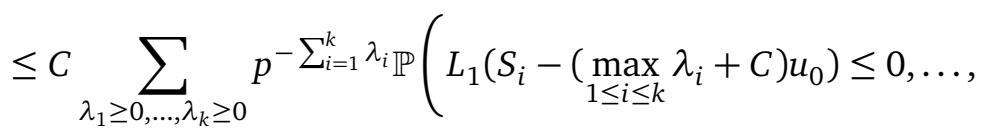

$$
\begin{aligned}
& \left.L_{k}\left(S_{i}-\left(\max _{1 \leq i \leq k} \lambda_{i}+C\right) u_{0}\right) \leq 0, \quad 0 \leq i \leq n ; \quad S_{n} \in K\right) .
\end{aligned}
$$

Choosing $C>0$ large enough, we can apply Theorem 4 of [26] and we deduce

$$
\begin{aligned}
\varphi_{n+1}(e) & \leq \frac{C}{n^{1+\frac{\sqrt{(l-2)^{2}+4 \lambda}}{2}}} \sum_{1 \geq 0, \ldots, \lambda_{k} \geq 0}\left(\max _{1 \leq i \leq k} \lambda_{i}+1\right)^{C} p^{-\sum_{i=1}^{k} \lambda_{i}} \\
& \leq \frac{C}{n^{1+\frac{\sqrt{(l-2)^{2}+4 \lambda}}{2}}} \sum_{1 \geq 0, \ldots, \lambda_{k} \geq 0}\left(\sum_{i=1}^{k} \lambda_{i}+1\right)^{C} p^{-\sum_{i=1}^{k} \lambda_{i}} \\
& \leq \frac{C}{n^{1+\frac{\sqrt{(l-2)^{2}+4 \lambda}}{2}}} .
\end{aligned}
$$

This completes the proof of Theorem 2.

\section{References}

[1] G. Alexopoulos. A lower estimate for central probabilities on polycyclic groups. Canad. J. Math. 44 (1992), 897-910. MR1186471

[2] G. Bachman. Introduction to p-Adic Numbers and Valuation Theory. Academic Press, 1964. MR0169847

[3] A. Borel, J. Tits. Groupes réductifs. Publications Math. IHES 27 (1965), 55-150. MR0207712

[4] J. W. S. Cassels. Local Fields. Camb. Univ. Press, 1986. MR0861410

[5] C. Chevalley. Théorie des Groupes de Lie, tome III. Hermann, 1955. MR0068552

[6] Th. Coulhon, A. Grigor'yan. On diagonal lower bounds for heat kernels on non-compact manifolds and Markov chains. Duke Math. J. 89 (1997), 133-199. MR1458975

[7] Th. Coulhon, A. Grigor'yan, Ch. Pittet. A geometric approach to on-diagonal heat kernel lower bounds on groups. Ann. Inst. Fourier 51 (2001), 1763-1827. MR1871289 
[8] R.I. Grigorchuk. Degrees of growth of finitely generated groups and the theory of invariant means. Izv. Akad. Nauk SSSR, Ser. Mat 48 (1984), 939-985. MR0764305

[9] Y. Guivarc'h. Croissance polynomiale et périodes des fonctions harmoniques. Bull. Soc. Math. France 101 (1973), 333-379. MR0369608

[10] W. Hebisch. On heat kernels on Lie groups. Math. Zeit. 210 (1992), 593-605. MR1175724

[11] W. Hebisch, L. Saloff-Coste. Gaussian estimates for Markov chains and random walks on groups. Annals of Probability 21 (1993), 673-709. MR1217561

[12] N. Jacobson. Lie Algebras, Interscience Publishers, 1962. MR0143793

[13] J. Jenkins. Growth of connected locally compact groups. J. Funct. Anal. 12 (1973), 113-127. MR0349895

[14] S. Mustapha. Random walks on unimodular p-adic groups. Stochastic Process and their Applications 115, (2005) 927-937. MR2134485

[15] S. Mustapha. Bornes inférieures pour les marches aléatoires sur les groupes $p$-adiques moyennables. Ann. Inst. H. Poincaré Probab. Statist. 42 (2006), 81-88. MR2196972

[16] Ch. Pittet, L. Saloff-Coste. On random walks on wreath products. Annals of Probability, 30 (2002), 948-977. MR1905862

[17] Ch. Pittet, Saloff-Coste. Random walks on finite rank solvable groups. Jour. Eur. Math. Soc. 4 (2003), 313-342. MR2017850

[18] C. R. E. Raja. On classes of p-adic Lie groups. New York J. Math 5 (1999), 101-105. MR1703206

[19] H. Reiter. Classical Harmonic Analysis and Locally Compact Groups. Oxford, Math. Monograph, 1968. MR1802924

[20] J.-P. Serre. Lie Algebras and Lie Groups. Benjamin, New York, 1965. MR0218496

[21] M. H. Taibleson. Fourier analysis on local fields. Princeton University Press, Princeton, N.J.; University of Tokyo Press, Tokyo, 1975. MR0487295

[22] N. Th. Varopoulos. Random walks on soluble groups. Bull. Sci. Math. 107 (1983), 337-344. MR0732356

[23] N. Th. Varopoulos. Groups of superpolynomial growth. Harmonic analysis (Sendai, 1990), ICM Satellite Conference Proceedings, Springer, 1991. MR1261441

[24] N. Th. Varopoulos. Diffusion on Lie groups I, II, III. Canad. J. Math. 46 (1994), 438-448, 1073-1092; 48 (1996), 641-672. MR1271225, MR1295132, MR1402333

[25] N. Th. Varopoulos. Wiener-Hopf theory and nonunimodular groups. J. Func. Analysis 120 (1994), 467-483. MR1266317

[26] N. Th. Varopoulos. Potential theory in conical domains. Math. Proc. Cambridge Philos. Soc. 125 (1999), 335-384. MR1643806 
[27] N. Th. Varopoulos. Marches aléatoires et diffusions dans les domaines lipschitziens. C. $R$. Acad. Sci. Paris Sér. I Math 332 (2001), 357-360. MR1821476

[28] N. Th. Varopoulos, L. Saloff-Coste, Th. Coulhon. Analysis and geomety on groups. Cambridge tracts in mathematics, 102, 1993. MR1218884 